\title{
Performance analysis of Compressive Modulation scheme in Digital Communication
}

\author{
Lokendra Singh $^{1}$, Anuj Sharma ${ }^{2}$, Dr.Sandeep Sharma ${ }^{3}$ \\ ${ }^{I}$ Department of Electronics \& Communication Engineering, DIT, Dehradun, India \\ ${ }^{2}$ Assistant Professor, Department of Electronics \& Communication Engineering, DIT, Dehradun, India \\ ${ }^{2}$ Associate Professor, Department of Electronics \& Communication Engineering, DIT, Dehradun, India
}

\begin{abstract}
Wireless communication system, gives good performance only when the selection of modulation process is best. Generally, to chose the particular modulation schemes different parameters are taken into consideration i.e. bandwidth efficiency, bit error rate, signal to noise ratio, cost, effectiveness and easy implementation, but since from very long time bandwidth efficiency is that parameter which plays important role in choosing any modulation scheme. As the waveform of all existing modulation scheme is separated in time domain which makes it difficult to improve their bandwidth efficiency. Since, from last decade researchers are continuously trying to overcome this problem and finally, they were able to discover such a technique which is able to reconstruct the original signal form aliasing environment named as compressive modulation (CM). The working principle of CM has a key part called compressive sensing, by the help of this former one is able to have larger bandwidth efficiency than our modulation techniques. In this paper, we proposed superior Compressive modulation technique and its comparative analysis with QPSK in regard of bandwidth efficiency and BER. The simulation results and theoretical analysis shows that CM has greater bandwidth than QPSK even at high as well as low SNR.
\end{abstract}

Keywords: Compressive Modulation, Compressive Sensing, QPSK.

\section{Introduction}

As the digital modulation schemes have many advantages over analog schemes, hence in our wireless communication system we use former modulation schemes. There are two main factors i.e. bandwidth efficiency and power efficiency, based on which the performance of digital modulation schemes is measured [1]. The modulation techniques which are commonly used in digital communication are ASK (Amplitude Shift Keying) PSK (Phase Shift Keying), FSK (Frequency Shift Keying), OFDM (Orthogonal Frequency Division Multiplexing) etc. [2]. Researchers are always struggling in improving their bandwidth efficiency during modulation. As the waveform of our modulation schemes is separated in time domain which makes it difficult to improve their bandwidth efficiency. While in the current time it is become very essential to attained higher bandwidth efficiency.

Our researcher are continuously work for attaining the higher bandwidth and finally they were able to proposed such a scheme which has higher bandwidth than our existing modulation schemes even at low SNR called Compressive Modulation (CM). The key part of CM is Compressive Sensing which says that during modulation process the code element which contains information, of our modulation schemes i.e. 4-PSK can be aliased in time domain.

While, aliased signal can be obtained by the product of code element and sinusoidal carrier wave, and then adding results with next forward shifted code for a portion of carrier wave period and so on. Compressing and adding all the code elements gives a aliased modulated signal in time domain. According to CS theory, original signal can be reconstructed from the few samples. By the help of CS theory it is also possible to capture and represent the lower rate than NQUIST rate. CS theory is also able to deal with sparse and under sampled signals even when they contains noise.

Compressive modulation is arising from the combination of digital modulation technique with Compressive Sensing. Earlier, CM combines CS theory with traditional QPSK and whose results show that former one has greater bandwidth than later one scheme. The basic key principle of compressive modulation is that, the original signal can be reconstructed at receiver using optimization but only when the transmitted signal is aliased during modulation at transmitter.

In this paper, detail description of BPSK, QPSK and CM is given in section II, III, \& IV respectively.

\section{BPSK (Binary Phase Shift Keying)}

The main modulation method used in present days is BPSK. Main key feature of this scheme is that it use the phase of a carrier wave to carry and transmit digitized information with keeping its amplitude and 
frequency remains unchanged. In general, the symbol " 0 " and " 1 " are represented by the phase of " $\pi$ " " 0 ", respectively. Hence, the BPSK signal in time domain is given as follows:

$$
\mathrm{e}_{\mathrm{BPSK}}(\mathrm{t})=\mathrm{s}_{\mathrm{o}}(\mathrm{t}) \cos \omega_{\mathrm{c}} \mathrm{t},
$$

Where, $\cos \omega_{c} t$ is sinusoidal carrier wave and $s(t)$ is bipolarity $100 \%$ duty ratio rectangular pulse sequence.

$$
\mathrm{s}_{0}(\mathrm{t})=\left\{\begin{array}{cc}
1 & \text { probability } \mathrm{P}, \text { code element " } 1 " \\
-1 & \text { probability } 1-\mathrm{P}, \text { code element }
\end{array}\right.
$$

After modulation the information carrying band-pass signals transmitted through channels, and original signal is restore at the receiving end using coherent demodulation. The main limitation in this scheme is that, the modulated signal is separated in time domain, due to which it become difficult to increase the transmission rate unless we increase the baseband frequency. This problem is solved using CM.

\section{QPSK (Quadrature Phase Shift Keying)}

The digital data (zeros and ones) is send to receiver from transmitter by the help of digital modulation schemes. In wireless channel symbols are represented in the form of Zeros and ones. In PSK, the phase of a carrier is varied between two or more states to represent zeros and ones. If the phase shift between two states is $180^{\circ}$, the modulation is called BPSK. While, when phase shift between two states become $90^{\circ}$, the modulation is called QPSK. In QPSK, the data transmission is occurs when the phase of original signal is modulated by $90^{\circ}$.

The bandwidth efficiency of different PSK is shown in table no. 1.

Table1. Bandwidth efficiency

\begin{tabular}{|c|c|c|c|c|c|c|}
\hline PSK Schemes & BPSK & QPSK & $\begin{array}{c}\text { 8- } \\
\text { PSK }\end{array}$ & $\begin{array}{c}\text { 16- } \\
\text { PSK }\end{array}$ & $\begin{array}{c}\text { 32- } \\
\text { PSK }\end{array}$ & $\begin{array}{c}\text { 64- } \\
\text { PSK }\end{array}$ \\
\hline $\begin{array}{c}\text { No. of bits } \\
\text { per symbol }\end{array}$ & 1 & 2 & 3 & 4 & 5 & 6 \\
\hline $\begin{array}{c}\text { Bandwidth } \\
\text { efficiency }\end{array}$ & 0.5 & 1 & 1.5 & 2 & 2.5 & 3 \\
\hline
\end{tabular}

In QPSK, we have two channels through which one bit is transmitted simultaneously and hence we have four states i.e.

$$
2^{\mathrm{M}}=2^{2}=4
$$

These two bits are encoded using Gray Code to minimize the Bit Error Rate. When the symbol ' 1 ' is transmitted, the average normalized power is given by the scaling factor $\sqrt{\frac{E_{s}}{2}}$, when all the constellation points are equally likely. The implementation of QPSK is more general than BPSK as well as higher order PSK. The symbols in constellation diagram are used to transmit the signal in terms of sine and cosine waves as,

Where $\mathrm{n}=1,2,3,4 \ldots \ldots$

$$
S_{n}(t)=\sqrt{\frac{2 E_{b}}{T_{s}}} \cos \left(2 \pi f_{c} t+(2 n-1) \frac{\pi}{4}\right.
$$

This yields four phase $\pi / 4,3 \pi / 4,5 \pi / 4$ and $7 \pi / 4$ as per our need. The results in a two-dimensional signal with unit basis function is given as,

$$
\begin{aligned}
& \emptyset_{1}(t)=\sqrt{\frac{2}{T_{s}}} \cos \left(2 \pi f_{c} t\right) \\
& \emptyset_{2}(t)=\sqrt{\frac{2}{T_{s}}} \cos \left(2 \pi f_{c} t\right)
\end{aligned}
$$

The first basis function is used as the in-phase component of the signal and the second as the quadrature component of the signal as shown in eq. 3 and 4 respectively. Hence, the signal constellation is consist of 4 signal space points as follows,

$$
\pm \sqrt{\mathrm{E}_{\mathrm{b}} / 2} \pm \sqrt{\mathrm{E}_{\mathrm{b}} / 2}
$$

Total power is equally divided between two carriers which is represented by the factor $1 / 2$.

\section{II.1.Parameters used for choosing modulation scheme.}

A) Power Efficiency is the ability of a modulation technique to conserve the delity (acceptable BER) of the digital message even at low power levels. It is often expressed as the ratio of Energy per bit to noise power 
$\left(E_{b} / N_{o}\right) \cdot E_{b} / N_{o}$ is the energy per bit to noise power spectral density ratio. It is important parameter during selection of a modulation scheme.

B) Bandwidth Efficiency is the efficient utilization of allocated bandwidth and defined as the throughput data rate per Hertz. Spectrum is expensive resource in wireless communication. Therefore, bandwidth efficient modulation schemes are always preferred.

C) Bit Rate $\left(\mathrm{R}_{\mathrm{b}}\right)$ is defined as the rate at which information is passed from transmitter to receiver. For QPSK bit rate is given as

$$
P_{S}=1-\left(1-P_{b}\right)^{2}
$$

D) Baud Rate is defined as the number of symbols/second. Each symbol represents $\mathrm{n}$ bits, and has $\mathrm{M}$ signal states, where $\mathrm{M}=2_{\mathrm{n}}$ and this is also known as M-ary signaling.

E) Bit Error Rate (BER) is the ratio of number of bit errors to the total number of transferred bits during a particular time interval. BER is a unit less performance measure, often expressed as a percentage. The bit error rate of QPSK is given as

$$
P_{b}=Q\left(\sqrt{\frac{2 E_{b}}{N_{o}}}\right)
$$

F) Signal to Noise Ratio (SNR) is defined as the ratio between signal to noise power and normally expressed in decibel $(\mathrm{dB})$.

For QPSK SNR is given as

$$
\mathrm{P}_{\mathrm{S}} \approx 2 \mathrm{Q}\left(\sqrt{\frac{\mathrm{E}_{\mathrm{S}}}{\mathrm{N}_{0}}}\right)
$$

G) Additive White Gaussian Noise (AWGN) the additive means the noise is superimposed to the signal that tends to obscure or mask the signal where it will limit receiver ability to make correct symbol decisions and limits the rate of information transmission.

H) Rayleigh Fading. The Rayleigh distribution is commonly used to describe the statistical time varying nature of received envelope of flat fading signal or the envelope of individual multi-path component.

\section{Compressive Modulation.}

In this paper we combine QPSK with compressive sensing to compare both in terms of bandwidth efficiency. The key of Compressive Modulation is Compressive Sensing which is it's modulation scheme.

\section{IV.1. Modulation scheme}

CS theory says that, a signal is treated as aliased if it is sparse in time domain, and then original signal is reconstructed from that aliased signal by the help of optimization. Hence, in this theory we make modulated signal aliased in time domain in order to improve its bandwidth. Compressive sensing is different than the classical sampling in following two ways:

1) CS systems typically acquire measurements in the form of inner products between the signal and more general test functions while in other sensing sampling is done at specific points in time. The randomness often plays a key role in the design of these test functions.

2) The two frameworks differ in the sense that they deal with signal recovery, i.e., the problem of recovering the original signal from the compressed measurements.

CS has already had noticeable impact on several applications. One of them is medical imaging, where it has enabled speedups by a factor of seven in pediatric MRI while preserving diagnostic quality. CS theory also states that an d-dimensional signal having sparse or compressible representation can be reconstructed from ' 1 ' linear measurements even if $1<\mathrm{d}$.[3]. The acquisition of CS based signal is much better than simple sampling for sparse or compressible signal. Let us consider that $\mathbf{u}(\mathbf{t})$ is a analog signal which is sampled at NQUIST rate and processed with $S$ sample in frames. Each frame is $\mathbf{S} \times \mathbf{1}$ vector, ' $u$ ' is given as $\mathbf{u}=$ $\boldsymbol{\Psi S}$, where $\boldsymbol{\Psi}$ is an $\mathbf{S} \times \mathbf{S}$ matrix whose columns are similarly sampled with basis function ' $\mathbf{u}$ ' given by $\mathbf{u}=\mathbf{Z}$, where $\boldsymbol{\Psi}$ is an S matrix whose $\boldsymbol{\Psi}(\boldsymbol{t})$, and $\mathbf{Z}$ is the vector that chooses the linear combination of the basis function, $\mathbf{Z}$ is treated as that ' $\mathbf{u}$ ' is in domain of $\mathbf{\Psi}, \mathbf{Z}$ is considered as sparse signal for CS for its well performance. $\mathbf{Z}$ is considered as $\boldsymbol{K}$-sparse if it contains only $\boldsymbol{K}$ non-zero elements.

According to CS theory ' $\mathbf{u}$ ' can be reconstructed successfully from $\mathbf{M}$ measurements, where $\mathbf{M} \ll \mathbf{S}$ the measurement can be achieved by projecting $\mathbf{v}$ over another random basis $\boldsymbol{\Phi}$ that is incoherent with $\boldsymbol{\Psi}$. Thus representation of measurement of $\mathbf{M} \times \mathbf{1}$ vector $\mathbf{z}$ given by 


$$
\mathrm{z}=\Phi_{\mathbf{u}}=\Phi \Psi Z
$$

This is right CS signal for signal reconstruction, CS theory also uses convex optimization algorithm. The time delay between adjacent elements is determined by sampling at receiving end.

Hence, the equation (3) can also written as,

$$
\mathbf{z}=\sum_{\mathrm{i}=1}^{\mathrm{d}} \mathbf{u}(\mathbf{i}) \boldsymbol{\phi}_{\mathbf{i}}
$$

Where, $\mathbf{u}(\mathrm{i})$ is projection of measurement vecto $\mathbf{z}$ in dictionary $\boldsymbol{\Phi}$. Let the period of original code element signal is $\mathrm{P}$, then the bandwidth of $\mathrm{CM}$ will be $\mathrm{P} / \mathrm{t}_{\mathrm{x}}$ times of QPSK modulation.

\section{IV.2. Optimization and Reconstruction of original code element}

The process of getting the original code element stream is as follows,

$$
\min _{\mathrm{u}}\|\mathrm{u}\|_{0} \quad \text { s. t. } \mathbf{z}=\boldsymbol{\Phi}_{\mathbf{u}}
$$

While, the equation (5) is non-convex optimization problem. According to CS theory if dictionary $\boldsymbol{\Phi}$ is in-coherent, then the model of $\mathrm{l}_{0}$ norm is given as,

$$
\min _{\mathrm{u}}\|\mathrm{u}\|_{0} \text { s. t. } \mathbf{z}=\boldsymbol{\Phi}_{\mathbf{u}}
$$

In this way the problem becomes convex and can be mitigate via existing algorithms. Let us consider that channel is AWGN (Additive White Gaussian Noise) channel, and received signal is expressed as,

$$
\mathrm{z}_{\mathrm{d}}=\mathrm{z}+\mathrm{b}
$$

Where, $\mathrm{b}$ is noise in the channel, following the normal distribution $\mathbf{b} \sim \mathbf{N}\left(\mathbf{0}, \boldsymbol{\sigma}^{2}\right)$. Incoherence of $\boldsymbol{\Phi}$ is,

$$
\hat{u}=\underset{\mathrm{u}}{\arg \min }\left\|\mathrm{z}_{\mathrm{d}}-\boldsymbol{\Phi}_{\mathrm{u}}\right\|_{2}^{2}+\mu\|\mathrm{u}\|
$$

Where $\mu$ is a weight factor. Optimization result is only obtained when $\mu$ is,

$$
\mu=\sigma \sqrt{2 \log d}
$$

Where, $\mathrm{d}$ refers to the length of ' $\mathbf{u}(\mathbf{t})$ ', and $\sigma$ can be determined by the received noise signal when no signal is transmit by the transmitter. By using SVD the influence of noise signal on received signals at reconstructive results is minimized. According to the Principle Component Analysis (PCA) theory dictionary of $\Phi$ is,

$$
\Phi=\mathrm{V}_{\Phi} \Lambda_{\Phi} \mathrm{C}_{\Phi}^{\mathrm{P}}
$$

$\boldsymbol{\Phi}$ can be represented approximately by the linear component of first $\mathbf{k}$ principle component of it. i. e.

The parameter can be calculated as

$$
\mathrm{B}=\mathrm{V}_{\boldsymbol{\Phi}, \mathbf{k}} \Lambda_{\boldsymbol{\Phi}, \mathbf{k}} \mathrm{V}_{\boldsymbol{\Phi}, \mathbf{k}}^{\mathrm{P}}
$$

$$
\widehat{\mathrm{k}}=\underset{\mathrm{k}}{\arg \min }\left|\frac{\sum_{\mathrm{i}=1}^{\mathrm{k}} \lambda_{\mathrm{i}}^{2}}{\sum_{\mathrm{i}=\mathrm{k}+1}^{\mathrm{n}} \lambda_{\mathrm{i}}^{2}}-\frac{\mathrm{e}_{\mathrm{u}}}{\mathrm{e}_{\mathrm{d}}}\right|
$$

Where, $\mathrm{e}_{\mathrm{u}}$ and $\mathrm{e}_{\mathrm{d}}$ are total energy of modulated signal and noise. The speed of reconstruction algorithm is improved by reducing the scale of $\boldsymbol{\Phi}$, and respectively the length of measurement vector $\mathrm{z}$ is shortened, as follows:

$$
\omega=\mathrm{V}_{\boldsymbol{\Phi}, \mathbf{k}} \mathrm{V}_{\boldsymbol{\Phi}, \mathbf{k}}^{\mathrm{P}} \mathrm{Z}
$$

Where, $\omega$ is a low noise estimation of $z$ since the first $k$ principle component in $\omega$ have fewer noises than original received signal $\mathrm{z}$ has. The optimization model in equation (8) is expressed as,

$$
\hat{\mathrm{u}}=\underset{\mathrm{u}}{\arg \min }\|\omega-\mathrm{Bu}\|_{2}^{2}+\mu\|\mathrm{u}\|
$$

A good estimation of $\mathrm{v}$ in which we have position information of code elements " 1 "s can be obtained faster and accurately. Hence, the original code element stream is reconstructed by using $3 \sigma$-rule.

\section{IV.3. Spectral Analysis of Compressive Modulated Signal}

In communication system, the bandwidth is very important parameter, which often means higher cost communication. 
Let us consider that $b_{i}(t)=(1,2 \ldots d)$ is carrier wave form sequence correspond to $\phi_{i}(1,2 \ldots d)$, in Ф. it is all zeros but period of code element with different amounts of shift in time domain, then the modulated signal can be formulated as,

$$
\mathrm{e}_{\mathrm{CM}}(\mathrm{t})=\sum_{\mathrm{i}=1}^{\mathrm{d}} \mathrm{Z}(\mathrm{t}) \mathrm{b}_{\mathrm{i}}(\mathrm{t})
$$

Where, $\mathrm{Z}_{0}(\mathrm{t})$ is representing code element stream in time domain and $\mathrm{d}$ is number of wave atoms in $\boldsymbol{\Phi}$. Length of $b_{i}(t)$ and $e_{C M}(t)$ is

$$
\mathrm{L}=(\mathrm{d}-1) \mathrm{t}_{\mathrm{u}}+\mathrm{P}
$$

Where, $t_{U}$ is set of $\mathrm{P}$ and $\mathrm{L}$ is equal to $\mathrm{St}$ and $\mathrm{e}_{\mathrm{CM}}(\mathrm{t})$ is same as QPSK modulated signal, which is given as

$$
\eta=\frac{1}{(\text { Bandwidth } \times \text { Symbol period })}=\frac{P_{f}}{P}
$$

Where, $\mathrm{P}$ is symbol period and $\mathrm{P}_{\mathrm{f}}$ is approx. bandwidth of QPSK. According to Linear Theorem of Fourier Transform, spectral characteristics of $\mathrm{e}_{\mathrm{CM}}(\mathrm{t})$ and QPSK modulated signal are similar. The above process shows that CM will not broadened the bandwidth of modulated signal due to which it is able to improve the bandwidth efficiency. Let the bandwidth of CM is $\eta_{\mathrm{CM}}$ and of QPSK is $\eta_{0}$ then the relationship between $\eta_{\mathrm{CM}}$ and $\eta_{0}$ is,

$$
\eta_{\mathrm{CM}}=\frac{\mathrm{s}}{\mathrm{t}_{\mathrm{s}}} \eta_{0}
$$

\section{Simulation and Experiment}

This section, tells about the performance of QPSK in AWGN channel and estimation that we made which is that, the maximum possible bandwidth efficiency under exactly same channel between CM and QPSK under certain BER values in same additive white Gaussian noise channel.
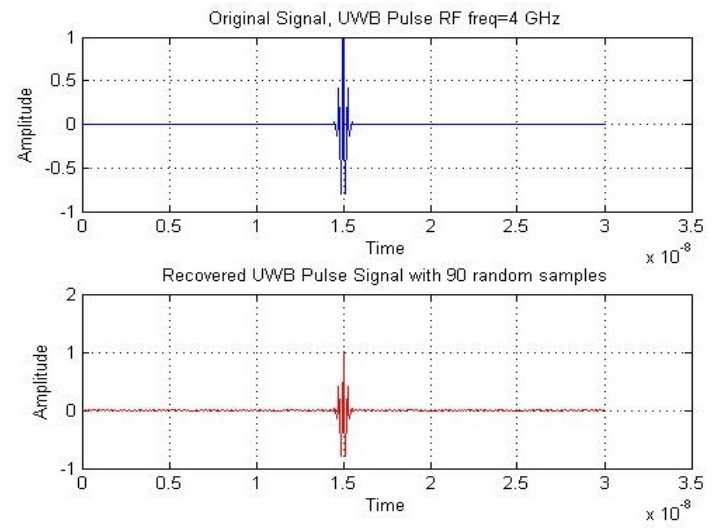

Fig.1.Original UWB signal at receiving end with 90 random samples
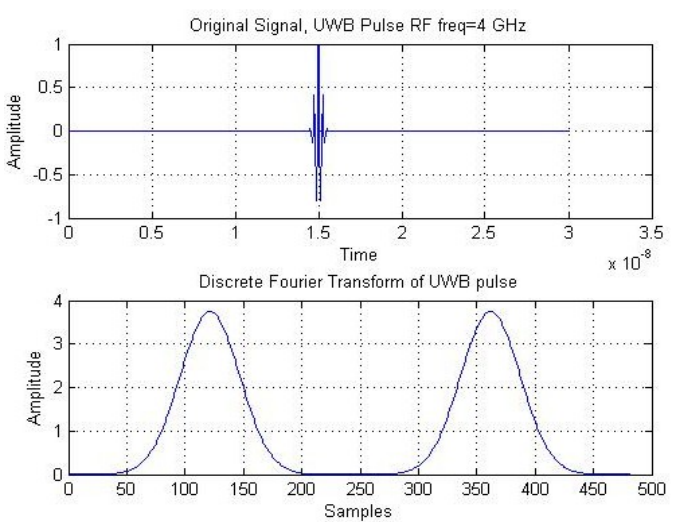

Fig.2. Discrete Fourier Transform of UWB signal

At the receiving end the SNR of modulating signal is from 2-20 dB. 


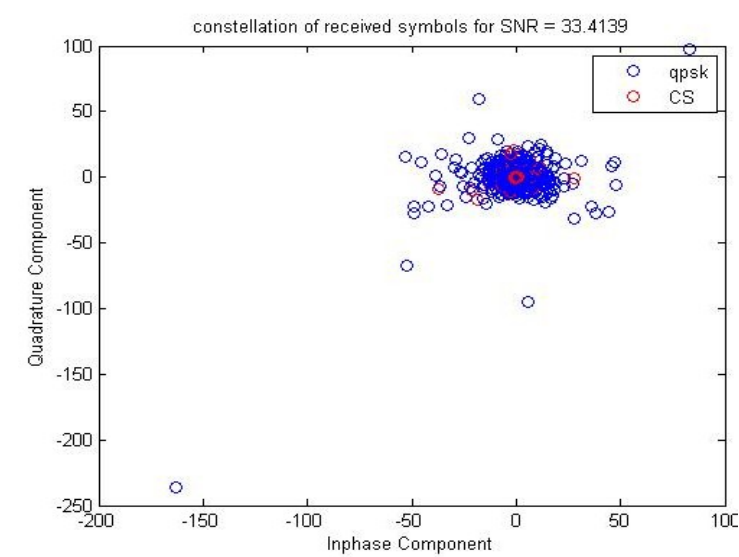

Fig.3. Constellation diagram of CM and QPSK at $\mathrm{SNR}=33.4139$

Baseband frequency $f_{s}$ is set to $15 \mathrm{kHz}$, and the sampling rate is set to $150 \mathrm{f}_{\mathrm{s}}$. The solver we used in the reconstructing process of CM is cvx. Usually, a communication process is considered as valid only when its BER is set to at least in an order of 10-4. Hence, throughout our code element transmission for achieving maximum possible bandwidth we estimate the transmission rate at different SNR levels of CM and QPSK at receiving end with a BER in an order of magnitude 10e-4. Comparison result is shown in figure given below

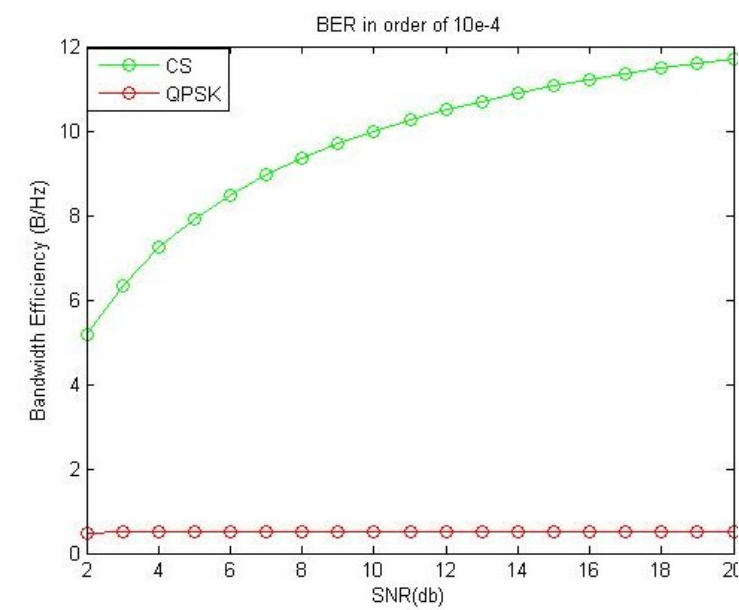

Fig.4. Comparison of code element transmission rate Between CM and QPSK (BER in order of $10^{-4}$ )

From the above results, it is seen that $\eta_{0}=1 \mathrm{~B} / \mathrm{Hz}$, and $\eta_{\mathrm{CM}}$ can be 12 times of $\eta_{0}$ at most and 5 times at least under the precondition of ensuring the reliability of communication. However, the BER of QPSK is calculated as follows;

$$
\operatorname{BER}=\frac{1}{2} \operatorname{erfc}(\sqrt{\mathrm{r}})
$$

Where, $r$ is the SNR of received signal. Meanwhile, for QPSK modulation schemes, if the code element transmission rate is increased, the channel bandwidth also increased, while CM will not occupy more channel bandwidth. Hence, it is convinced that CM scheme highly improves the bandwidth efficiency.

Even CM shows that it has good bandwidth efficiency but it has also some problems which should be improved in future works. Firstly, the optimization algorithm used at the receiving end takes more time, which is common drawback of CS. In this paper we reconstruct the original signal by dividing the signal into many samples to save the time. Secondly, the sampling rate at the receiving end of CM is much higher than that of QPKS. Although, the CM performs well even at low SNR, but it still needs to improved at high SNR for better reliability of communication systems and this problem can be mitigate by using the effective error control coding.

\section{Conclusion And Future Works}

In this paper, with the guidance of CS theory we ameliorate the traditional QPSK and propose a new modulation scheme, CM, which highly improves the bandwidth efficiency in digital communication system. By, 
the effectiveness of this method, it can be applied to other existing modulated schemes like MPSK, OFDM and so on in future. The experiment results confirm the above conclusion.

\section{ACKNOWLEDGEMENT}

It was my great pleasure in extending special thanks to Mr. Anuj Sharma and my family under whose guidance and help, I was able to propose my work in the form of this research paper. I' am deeply inhibited to themselves for their training and guidance throughout my work. They was always been a source of constant inspiration and encouragement for me.

\section{References}

[1] Bernard sklar, 2011. Digital communications: fundamentals and applications. Prentice Hall, 277-281.

[2]. Bernard Sklar, 2011. Digital communications: Fundamentals and Applications. Prentice Hall, $277-281$.

[3] R.Calderbank, S. Jafarpour and R. Schapire. 2009. Compressed learning: universal uparse dimensionality reduction and learning in the measurement domain.

[4] B. Sklar, 2011. Digital Communications-Fundamentals And Applications, 2nd, New Jersey: Prentice Hall.

[5] L. Donoho, 2006. Compressed sensing, IEEE Transactions on Information Theory, vol. 52, no. 4, pp. 1289-1306.

[6] G. Baraniuk, 2007. Compressive Sensing, IEEE Signal Processing Magazine, vol. 24, no. 4, pp. 118-121.

[7] J. Candes and B. Wakin, 2008. An introduction to compressive sampling, IEEE Signal Processing Magazine, vol. 25, no. 2, pp. 21-30.

[8] J. Candes, J. Romberg and T. Tao, 2006. Robust uncertainty principles: Exact signal reconstruction from highly incomplete frequency information, IEEE Transactions on Information Theory, vol. 52, no. 2, pp. 489-509.

[9] J. Candes and J. Romberg, 2006. Quantitative Robust Uncertainty Principles and Optimally Sparse Decompositions, Foundations of Computational Mathematics, vol. 6, no. 2, pp. 227-254.

[10] G. Shi, C. Chen, J. Lin, X. Xie, and X. Chen, 2012. Narrowband Ultrasonic Detection with High Range Resolution: Separating Echoes via Compressed Sensing and Singular Value Decomposition, IEEE Transactions on Ultrasonics, Ferroelectrics and Frequency Control, vol. 59, no. 10, pp. 2237-2253.

[11] Z. Yu, S. Hoyos, and B.M. Sadlar, 2008.Mixed Signal parallel compressed sensing and reception for cognitive radio, in proc. IEEE Int. Conf on Acoustics. Speech and Signal Processing, Las Vegas, Nevads, USA.

[12] G. Shi, J. Lin, X. Chen, F. Qi, D. Liu, and Z. Li, 2008. UWB echo signal detection with ultra-low rate sampling based on compressed sensing, IEEE Transactions on Circuits and Systems II: Express Briefs, vol. 55, no. 4, pp. 379-383.

[13] S. Chen, L. Donoho, and A. Saunders, 1998. Atomic decomposition by basis pursuit, SIAM Journal on Scientific Computing, vol. 20, no. 1, pp. 33-61.

[14] I. Jolliffe, 2002. Principal Component Analysis, 2nd, Berlin: Springer- Verlag.

[15] A. Saleh and R. Valenzuela, A statistical model for indow multipath propagation, IEEEJ Select Areas Commun, vol.5, no.2, pp. $128-137$

[16] L. Jacques, J. Laska, P. Boufounos and R. Baraniuk. 2012. Robust 1-bit compressive sensing via binary _ stable embeddings of sparse vectors. Arxiv preprint arX- iv:1104.3160v2

[17] Y. Plan and R. Vershynin. 2011. One-bit compressed sensing by linear programming. Arxiv preprint arXiv:1109.4299.

[18] R. Calderbank, S. Jafarpour, and R. Schapire. 2009. Compressed learning: universal sparse dimensionally reduction and learning in the measurement domain.

\section{Appendices}

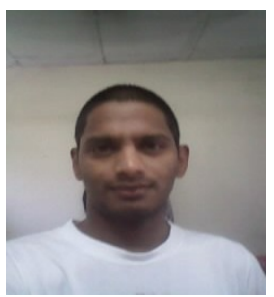

Lokendra singh presently lives in Dehradun and completed his Graduation as a B. Tech. graduate in the field of Electronics and communication from Uttar Pradesh Technical University, Lucknow, UP, India in 2012. He is a post-graduate scholar in the field of Wireless \& Mobile Communications from Dehradun Institute of Technology, Dehradun, Uttarakhand, India in 2013. He became the member of IJCSE and IJETT in 2014. Presently he is working on "Performance Analysis of Compressive Modulation in Digital Communication". 